\title{
Large bounded degree trees in expanding graphs
}

\author{
József Balogh* Béla Csabał Martin Pei ${ }^{\ddagger}$ and Wojciech Samotij ${ }^{\S}$
}

Submitted: Jun 2, 2009; Accepted: Dec 17, 2009; Published: Jan 5, 2010

Mathematics Subject Classification: 05C80, 05D40, 05C05, 05C35

\begin{abstract}
A remarkable result of Friedman and Pippenger [4] gives a sufficient condition on the expansion properties of a graph to contain all small trees with bounded maximum degree. Haxell [5] showed that under slightly stronger assumptions on the expansion rate, their technique allows one to find arbitrarily large trees with bounded maximum degree. Using a slightly weaker version of Haxell's result we prove that a certain family of expanding graphs, which includes very sparse random graphs and regular graphs with large enough spectral gap, contains all almost spanning bounded degree trees. This improves two recent tree-embedding results of Alon, Krivelevich and Sudakov [1].
\end{abstract}

\section{Introduction}

A very well-known folklore result on tree-embedding states that every graph with minimum degree at least $k$ contains all trees with at most $k$ edges and this is best possible (as illustrated by an arbitrarily large disjoint union of $(k+1)$-vertex complete graphs). A natural question arises - what additional assumptions on a graph can force it to contain certain trees? For an arbitrary graph $H$ and a set $X \subseteq V(H)$, let $N_{H}(X)$ denote the set of neighbors in $H$ of vertices in $X$. Extending a path-embedding result of Pósa [7],

*Department of Mathematics, University of California, San Diego, 9500 Gilman Drive, La Jolla, CA 92093, USA; and Department of Mathematics, University of Illinois, Urbana, IL 61801, USA. E-mail address: jobal@math.uiuc.edu. This material is based upon work supported by NSF CAREER Grant DMS-0745185 and DMS-0600303, UIUC Campus Research Board Grants 09072 and 08086, and OTKA Grant K76099

$\dagger$ Department of Mathematics, Western Kentucky University, Bowling Green, KY 42101, USA. E-mail address: bela.csaba@wku.edu. This research was partially supported by a New Faculty Scholarship Grant of WKU and by OTKA Grant K76099.

$\ddagger$ Department of Combinatorics and Optimization, University of Waterloo, Waterloo, ON, Canada. Email address: mpei@uwaterloo.ca.

$\S$ Department of Mathematics, University of Illinois, Urbana, IL, 61801, USA. Research supported in part by Trijtzinsky Fellowship and James D. Hogan Memorial Scholarship Fund. E-mail address: samotij2@illinois.edu. 
Friedman and Pippenger [4] proved that all graphs satisfying certain expansion properties contain all small trees with bounded maximum degree.

Theorem 1 ([4]). Let $m$ and $d$ be positive integers and let $H$ be a non-empty graph. Moreover, assume that every $X \subseteq V(H)$ with $|X| \leqslant 2 m$ satisfies $\left|N_{H}(X)\right| \geqslant(d+1)|X|$. Then $H$ contains every tree with $m$ vertices and maximum degree at most $d$.

An apparent shortcoming of Theorem 1 is that it can be helpful in finding only relatively small trees. Namely, in a graph of order $n$, the size of the largest tree the existence which is guaranteed by Theorem 1 is only about $n /(2 d+2)$, where $d$ is the maximum degree of the tree. Building on the ideas developed by Friedman and Pippenger [4], Haxell [5] managed to overcome this problem.

Theorem 2 ([5]). Let $T$ be a tree with $t$ edges and maximum degree $d$. Let $\emptyset=T_{0} \subseteq$ $T_{1} \subseteq \cdots \subseteq T_{\ell} \subseteq T$ be a sequence of subtrees of $T$ such that $T$ can be obtained by attaching new leaves to $T_{\ell}$. Let $d=d_{1} \geqslant \ldots \geqslant d_{\ell}$ be a sequence of integers such that for each $i$ with $1 \leqslant i \leqslant \ell$ and each $v \in V(T)$ we have

$$
\delta_{T}(v)-\delta_{T_{i-1}}(v) \leqslant d_{i},
$$

where $\delta_{S}(v)$ denotes the degree of $v$ in the subtree $S$ (if $v \notin V(S)$, then we let $\delta_{S}(v)=0$ ). Let $t_{i}=\left|E\left(T_{i}\right)\right|$. Suppose that $m \geqslant 1$ is an integer and $H$ is a graph satisfying the following conditions

1. For every subset $X \subseteq V(H)$ with $0<|X| \leqslant m,\left|N_{H}(X)\right| \geqslant d|X|+1$.

2. For every subset $X \subseteq V(H)$ with $m<|X| \leqslant 2 m$, and for each $i \in\{1, \ldots, \ell\}$, $\left|N_{H}(X)\right| \geqslant d_{i}|X|+t_{i}+1$.

3. For every subset $X \subseteq V(H)$ with $|X|=2 m+1,\left|N_{H}(X)\right| \geqslant t+1$.

Then $H$ contains $T$ as a subgraph. Moreover, for any vertex $x_{0}$ of $T_{1}$ and any $y \in V(H)$, there exists an embedding $f$ of $T$ into $H$ such that $f\left(x_{0}\right)=y$.

As an immediate corollary of the somewhat technical Theorem 2 , we derive the following statement.

Theorem 3. Let $d, m$ and $M$ be positive integers, and let $0 \leqslant L \leqslant 2 d m$. Assume that $H$ is a non-empty graph satisfying the following two conditions.

1. For every $X \subseteq V(H)$ with $0<|X| \leqslant m,\left|N_{H}(X)\right| \geqslant d|X|+1$.

2. For every $X \subseteq V(H)$ with $m<|X| \leqslant 2 m,\left|N_{H}(X)\right| \geqslant d|X|+M$.

Then $H$ contains every tree $T$ with $M+L$ vertices and maximum degree at most $d$, provided that $T$ has at least $L$ leaves. 
It turns out that Theorem 3 has a few very interesting and yet quite straightforward consequences. First of all, it gives a sufficient condition on the edge probability that almost surely forces the Erdös-Rényi random graph $G(n, p)$ to contain all nearly spanning bounded degree trees.

Theorem 4. Let $d \geqslant 2$ and $0<\varepsilon<1 / 2$. If

$$
c>\max \left\{1000 d \log (20 d), \frac{30 d}{\varepsilon} \log \frac{4 e}{\varepsilon}\right\},
$$

then the random graph $G(n, c / n)$ almost surely contains every tree with maximum degree $d$ and order at most $(1-\varepsilon) n$.

Theorem 4 significantly improves the ' $c \geqslant \frac{10^{6} d^{3} \log d \log ^{2}(2 / \varepsilon)}{\varepsilon}$ ' lower bound on the edge probability obtained by Alon, Krivelevich and Sudakov [1] with a lengthier and more complex argument making use of Theorem 1. Recently, in his doctoral thesis [6] the third author, using Theorem 2 and a refinement of the piece-by-piece embedding method from [1], obtained an improvement of the above mentioned result of Alon, Krivelevich and Sudakov [1] that is slightly weaker than Theorem 4. Note that in [1] it is suggested that in the statement of Theorem 4 the condition on the constant $c$ could be lowered to $\Theta(d \log (1 / \varepsilon))$. Finally, we would like to remark that a somewhat stronger version of Theorem 4 can be proved. In [3] it is shown that whenever $c$ is a large enough constant and $p \geqslant c / n$, then the local resilience (see, e.g., [8]) of the random graph $G(n, p)$ with respect to the property of containing all bounded degree almost spanning trees is almost surely $1 / 2+o(1)$.

For an $n$-vertex graph $G$, let $\lambda_{1}, \ldots, \lambda_{n}$ be the eigenvalues of its adjacency matrix, where $\lambda_{1} \geqslant \ldots \geqslant \lambda_{n}$. The second eigenvalue of $G$ is $\lambda(G):=\max _{i \geqslant 2}\left|\lambda_{i}\right|$. A graph $G$ is called an $(n, D, \lambda)$-graph if it is $D$-regular, has $n$ vertices and its second eigenvalue is at most $\lambda$. It is well-known that if $\lambda$ is much smaller than $D$, then $G$ has strong expansion properties. The following result, which is another consequence of Theorem 3 , shows that an $(n, D, \lambda)$-graph $G$ with large spectral gap ${ }^{1} D / \lambda$ contains all almost spanning trees with bounded degree.

Theorem 5. Let $d \geqslant 2$ and $0<\varepsilon<1 / 2$. If

$$
\frac{D}{\lambda}>\frac{\sqrt{8 d}}{\varepsilon}
$$

then every $(n, D, \lambda)$-graph contains all trees with maximum degree $d$ and order at most $(1-\varepsilon) n$.

Theorem 5 is again an improvement over the $\frac{D}{\lambda} \geqslant \frac{160 d^{5 / 2} \log (2 / \varepsilon)}{\varepsilon}$, lower bound obtained by Alon, Krivelevich and Sudakov [1].

\footnotetext{
${ }^{1}$ Although the spectral gap of a matrix is defined to be the difference between the moduli of its two largest eigenvalues, which in our setting is $D-\lambda$, the quantity $D / \lambda$, to which we refer to as the spectral gap, is a more natural measure of quasirandomness of $G$ in our considerations.
} 
Finally, the lower bounds on $c$ and $D / \lambda$ in Theorems 4 and 5 can be further improved if we restrict our attention to trees with large number of leaves.

Theorem 6. Let $d \geqslant 2,0<\varepsilon<1 / 2$ and $0<\lambda<1$. If

$$
c>\max \left\{1000 d \log (20 d), \frac{32 d}{\lambda} \log \frac{4 e}{\varepsilon}\right\},
$$

then the random graph $G(n, c / n)$ almost surely contains every tree with maximum degree $d$ and order at most $(1-\varepsilon) n$, provided that it has at least $\lambda n$ leaves.

Theorem 7. Let $d \geqslant 2,0<\varepsilon<1 / 2$ and $0<\lambda<1$. If

$$
\frac{D}{\lambda}>\sqrt{\frac{18 d}{\varepsilon \lambda}}
$$

then every $(n, D, \lambda)$-graph contains every tree with maximum degree $d$ and order at most $(1-\varepsilon) n$, provided that it has at least $\lambda n$ leaves.

The remainder of this note is organized as follows. In Section 2 we introduce a notion of graph expansion that gives rise to a certain family of expanding graphs, which we call $(\varepsilon, b, \alpha)$-expanders, and prove that under certain assumptions on the expansion parameters $\varepsilon, b$ and $\alpha$, every such graph contains all almost spanning bounded degree trees. The most technical (but standard) parts Sections 3 and 4, are entirely devoted to the study of expansion properties of random graphs and graphs with large spectral gap. Finally, in Section 5 , based on this study, we give very short proofs of our main results - Theorems 4 , 5,6 and 7 .

\section{Embedding trees in expanding graphs}

We start by defining a class of expanding graphs that seems to be most adequate and convenient in our further considerations.

Definition 8. Let $b \geqslant 2,0<\alpha<1$ and $0<\varepsilon<1 / b$. We will say that an $n$-vertex graph $G$ is an $(\varepsilon, b, \alpha)$-expander if it possesses the following two properties.

1. Every subset $X \subseteq V(G)$ of size at most $\varepsilon n$ satisfies $\left|N_{G}(X)\right| \geqslant b|X|$.

2. Every subset $X \subseteq V(G)$ of size at least $\varepsilon n$ satisfies $\left|N_{G}(X)\right| \geqslant(1-\alpha) n$.

As immediate consequences of Theorem 3 we derive the following sufficient conditions on the expansion parameters $\varepsilon, b$ and $\alpha$ which guarantee that all $(\varepsilon, b, \alpha)$-expanders contain every almost spanning tree with bounded maximum degree and, additionally, many leaves.

Corollary 9. Let $d \geqslant 2$ and $0<\varepsilon<1$. Suppose that $\alpha, \varepsilon_{0}>0$ are such that $2 d \varepsilon_{0}+\alpha \leqslant$ $\varepsilon$. Then every $n$-vertex $\left(\varepsilon_{0}, d+1, \alpha\right)$-expander contains all trees of order $(1-\varepsilon) n$ and maximum degree $d$. 
Proof. Let $G$ be an $n$-vertex $\left(\varepsilon_{0}, d+1, \alpha\right)$-expander. It is straightforward to check that $G$ satisfies assumptions of Theorem 3 with $m:=\varepsilon_{0} n, M:=\left(1-2 d \varepsilon_{0}-\alpha\right) n$ and $L:=0$. Hence $G$ contains every tree with maximum degree $d$ and order $M \geqslant(1-\varepsilon) n$.

Corollary 10. Let $d \geqslant 2$ and $0<\varepsilon, \lambda<1$. Then every $n$-vertex $(\lambda /(2 d), d+1, \varepsilon)$ expander contains all trees of order $(1-\varepsilon) n$ and maximum degree $d$ which contain at least $\lambda$ leaves.

Proof. Let $G$ be an $n$-vertex $(\lambda /(2 d), d+1, \varepsilon)$-expander. It is straightforward to check that $G$ satisfies assumptions of Theorem 3 with $m:=\lambda n /(2 d), L:=\lambda n$ and $M:=(1-\varepsilon-\lambda) n$. Hence $G$ contains every tree $T$ with maximum degree $d$ and order $M+L=(1-\varepsilon) n$, provided that $T$ has at least $\lambda n$ leaves.

\section{$3 \quad$ Expanding properties of random graphs}

For two not necessarily disjoint subsets of the set of vertices of a graph $G$, let

$$
e(A, B):=|\{(a, b) \in A \times B:\{a, b\} \in E(G)\}| .
$$

Lemma 11. Let $0<\beta \leqslant \gamma \leqslant 1 / 2$ and $c \geqslant \frac{3}{\beta} \log \frac{e}{\gamma}$. Then almost surely the random graph $G(n, c / n)$ does not contain two disjoint sets $B, C$ of size at least $\beta n$ and $\gamma n$ respectively, such that $e(B, C)=0$.

Proof. If $G(n, c / n)$ contains two sets $B$ and $C$ as in the statement of this lemma, clearly we can also find two disjoint sets $B^{\prime}$ and $C^{\prime}$ of size exactly $\beta n$ and $\gamma n$ respectively, with $e\left(B^{\prime}, C^{\prime}\right)=0$. The probability that such a pair exists is at most

$$
\left(\begin{array}{c}
n \\
\beta n
\end{array}\right)\left(\begin{array}{c}
n \\
\gamma n
\end{array}\right) \cdot\left(1-\frac{c}{n}\right)^{\beta \gamma n^{2}} \leqslant\left(\begin{array}{c}
n \\
\gamma n
\end{array}\right)^{2} \cdot e^{-c \beta \gamma n} \leqslant\left(\frac{e n}{\gamma n}\right)^{2 \gamma n} \cdot\left(\frac{e}{\gamma}\right)^{-3 \gamma n}=o(1) .
$$

Lemma 12. Let $0<\beta \leqslant \gamma \leqslant 1 / 2$ and let $c \geqslant \frac{6 \gamma}{\beta} \log \frac{e}{\gamma}$. Then almost surely $G(n, c / n)$ does not contain a pair of disjoint sets $B$ and $C$ of sizes at least $\beta n$ and at least $(1-\gamma) n$ respectively with $e(B, C)=0$.

Proof. As in the proof of Lemma 11, we only need to show that almost surely there is no such pair with sizes exactly $\beta n$ and $(1-\gamma) n$. The probability that such a pair exists is at most

$$
\left(\begin{array}{c}
n \\
\beta n
\end{array}\right)\left(\begin{array}{c}
n \\
(1-\gamma) n
\end{array}\right)\left(1-\frac{c}{n}\right)^{\beta(1-\gamma) n^{2}} \leqslant\left(\begin{array}{c}
n \\
\gamma n
\end{array}\right)^{2} \cdot e^{-c \beta n / 2} \leqslant\left(\frac{e n}{\gamma n}\right)^{2 \gamma n} \cdot\left(\frac{e}{\gamma}\right)^{-3 \gamma n}=o(1) .
$$

Lemma 13. Let $k \geqslant 2$ and let $c \geqslant 10 k \log _{2} k$. Then almost surely every subset $A$ of at most $n /($ ek) vertices in the random graph $G(n, c / n)$ spans less than $c|A| / k$ edges. 
Proof. Certainly, if a subset $A$ of size $a$ violates the assertion, $a \geqslant c / k$. The probability that there is a bad subset $A$ of size $a$, with $c / k \leqslant a \leqslant n / e k$, is at most

$$
\begin{aligned}
\left(\begin{array}{l}
n \\
a
\end{array}\right)\left(\begin{array}{l}
a^{2} / 2 \\
a c / k
\end{array}\right) \cdot\left(\frac{c}{n}\right)^{c a / k} & \leqslant\left(\frac{e n}{a}\right)^{a} \cdot\left(\frac{e a^{2}}{2} \cdot \frac{k}{a c}\right)^{c a / k} \cdot\left(\frac{c}{n}\right)^{c a / k} \\
& =\left(\frac{e n}{a} \cdot\left(\frac{e k a}{2 n}\right)^{c / k}\right)^{a} \leqslant\left(\frac{(e k / 2)^{c / k+1}}{(n / a)^{c / k-1}}\right)^{a} .
\end{aligned}
$$

If $\sqrt{n} \leqslant a \leqslant n / e k$, then

$$
\frac{(e k / 2)^{c / k+1}}{(n / a)^{c / k-1}} \leqslant\left(\frac{1}{2}\right)^{c / k} \cdot(e k)^{2} \leqslant k^{-10} \cdot(e k)^{2} \leqslant \frac{1}{2},
$$

and consequently (1) is bounded by $2^{-\sqrt{n}}$. In case $\left.c / k \leqslant a \leqslant \sqrt{(} n\right)$, (1) can be further estimated as follows

$$
\left(\frac{(e k / 2)^{c / k+1}}{(n / a)^{c / k-1}}\right)^{a} \leqslant\left(\frac{(e k / 2)^{11}}{(\sqrt{n})^{9}}\right)^{10}=o\left(n^{-1}\right) .
$$

Summing these estimates over all values of $a$ yields the desired result.

Lemma 14. Let $0<\rho<1 / 2$. If $c>64 \log \frac{e}{\rho}$, then almost surely the random graph $G(n, c / n)$ contains an induced subgraph $G^{\prime}$ with at least $(1-\rho) n$ vertices and minimum degree at least $c / 4$.

Proof. Let $G$ be our random graph $G(n, c / n)$. While $G$ contains a vertex with degree less than $c / 4$, delete that vertex. Denote the remaining induced subgraph of $G$ by $G^{\prime}$. If $G^{\prime}$ has at least $(1-\rho) n$ vertices, we have found the subgraph we were looking for. It suffices to show that the probability of $G^{\prime}$ having less than $(1-\rho) n$ vertices is small. First observe that if we were forced to delete more than $\rho n$ vertices, then the original graph $G$ contained a set $A$ of size $\rho n$ such that $e_{A}:=e(A, V(G)-A)<\rho c n / 4$. Note that $\mathbb{E}\left[e_{A}\right]=$ $\rho(1-\rho) c n \geqslant \rho c n / 2$. By standard Chernoff-type estimates (see, e.g., Theorem A.1.13 in [2]), the probability of this event in our random graph is at most

$$
\mathbb{P}\left(e_{A}<c \rho n / 4\right) \leqslant \mathbb{P}\left(e_{A}-\mathbb{E}\left[e_{A}\right]<-\rho c n / 4\right) \leqslant e^{-\frac{(\rho c n / 4)^{2}}{2 \rho c n}}=e^{-\rho c n / 32} .
$$

Hence the probability that such a set $A$ exists in our graph $G$ is bounded by

$$
\left(\begin{array}{c}
n \\
\rho n
\end{array}\right) \cdot e^{-\rho c n / 32} \leqslant\left(\frac{e n}{\rho n}\right)^{\rho n} \cdot\left(\frac{e}{\rho}\right)^{-2 \rho n}=o(1) .
$$

Theorem 15. Let $b \geqslant 2$ and $0<\rho \leqslant \varepsilon \leqslant \alpha<1 / 2$, where $\varepsilon<1 /(2 b+4)$. If

$$
c>\max \left\{500 b \log (12 b), \frac{6}{\varepsilon} \log \frac{2 e}{\alpha}, 64 \log \frac{e}{\rho}\right\},
$$

then almost surely the random graph $G(n, c / n)$ contains an induced subgraph $G^{\prime}$ of order at least $(1-\rho) n$ that is an $(\varepsilon, b, \alpha)$-expander. 
Proof. By Lemma 14, almost surely $G(n, c / n)$ contains an induced subgraph $G^{\prime}$ of order $n^{\prime}$, with $n^{\prime} \geqslant(1-\rho) n$ and $\delta\left(G^{\prime}\right) \geqslant c / 4$. Conditioning on that event, we will show that $G^{\prime}$ is almost surely an $(\varepsilon, b, \alpha)$-expander.

Suppose that $G^{\prime}$ fails to possess property 1 from Definition 8 . Then there is a set $X \subseteq V\left(G^{\prime}\right)$ of size $t$, with $t \leqslant \varepsilon n^{\prime}$ and $\left|N_{G^{\prime}}(X)\right| \leqslant b t$. Let $A:=X \cup N_{G^{\prime}}(X)$. Clearly $|A| \leqslant(b+1) t$. We consider three cases, depending on the order of $t$.

Case $1 . t \leqslant \frac{n}{8 e(b+1)^{2}}$.

Let $k:=8(b+1)$. Since edges incident to vertices in $X$ are contained in $A, e(A) \geqslant$ $\delta\left(G^{\prime}\right)|X| / 2 \geqslant c t / 8 \geqslant c|A| / k$. By our assumptions, $|A| \leqslant n /(e k)$, and $c>10 k \log _{2} k$. By Lemma 13, such non-expanding set $X$ almost surely does not exist.

Case 2. $\frac{n}{8 e(b+1)^{2}} \leqslant t \leqslant \frac{n}{20 e(b+1)}$.

Since $G^{\prime}$ is an induced subgraph, in $G$ there are no edges between $X$ and $Y:=V\left(G^{\prime}\right)-A$. By our assumptions on $t$ and $\varepsilon$, the latter set has at least

$$
n^{\prime}-|A| \geqslant(1-\rho) n-(b+1) t \geqslant n-n /(b+1)-(b+1) t \geqslant n-(8 e+1)(b+1) t
$$

vertices. Let $\beta:=t / n$ and $\gamma:=(8 e+1)(b+1) \beta$. By our assumption on $t$, we have that $\beta \geqslant \frac{1}{8 e(b+1)^{2}}$ and consequently $e / \gamma<12 b$. Moreover, note that $6 \gamma / \beta<500 b$. It follows that $c>6 \frac{\gamma}{\beta} \log \frac{e}{\gamma}$ and, by Lemma 12 , such non-expanding set $X$ almost surely does not exist.

Case 3. $\frac{n}{20 e(b+1)} \leqslant t \leqslant \varepsilon n^{\prime}$.

Again, in $G$ there are no edges between $X$ and $Y:=V\left(G^{\prime}\right)-A$. By our assumptions on $t$ and $\varepsilon$, the latter set has at least

$$
n^{\prime}-|A| \geqslant(1-(b+1) \varepsilon) n^{\prime} \geqslant(1-(b+1) \varepsilon)(1-\varepsilon) n \geqslant(1-(b+2) \varepsilon) n \geqslant \frac{n}{2}
$$

vertices. Let $\beta:=\frac{1}{20 e(b+1)}$ and $\gamma:=1 / 2$. Clearly $c>(3 / \beta) \log (e / \gamma)$. By Lemma 11 , such non-expanding set $X$ almost surely does not exist.

Hence almost surely the graph $G^{\prime}$ satisfies property 1 from definition 8. Finally, suppose that $G^{\prime}$ fails to possess the other property. Then there is a set $X$ of size exactly $\varepsilon n^{\prime}$ with $\left|N_{G^{\prime}}(X)\right| \leqslant(1-\alpha) n^{\prime}$. It follows that in $G$ there are no edges between $X$ and $Y:=V\left(G^{\prime}\right)-X-N_{G}(X)$. Clearly $Y$ contains at least $\alpha n^{\prime} \geqslant \alpha n / 2$ vertices. Let $\beta:=\varepsilon / 2$ and $\gamma:=\alpha / 2$. Since $c>(3 / \beta) \log \frac{e}{\gamma}$, by Lemma 11, this almost surely does not happen.

\section{Expanding properties of quasi-random graphs}

In [2], it is proved that for every two subsets $A$ and $B$ of the set of vertices of an $(n, D, \lambda)$ graph $G$,

$$
\left|e(A, B)-\frac{|A||B| D}{n}\right| \leqslant \lambda \sqrt{|A||B|}
$$


Theorem 16. Let $b \geqslant 2, \alpha>0$ and $0<\varepsilon<1 / b$. If

$$
\frac{D}{\lambda}>\max \left\{\frac{\sqrt{b}}{1-b \varepsilon}, \frac{1}{\sqrt{\alpha \varepsilon}}\right\}
$$

then every $(n, D, \lambda)$-graph $G$ is an $(\varepsilon, b, \alpha)$-expander.

Proof. Suppose that $G$ fails to possess property 1 from Definition 8 . Then there is a set $X \subseteq V\left(G^{\prime}\right)$ of size $t$, with $t \leqslant \varepsilon n$ and $Y:=N_{G}(X) \leqslant b t$. Since $G$ is $D$-regular, clearly $e(X, Y) \geqslant D t$. On the other hand, by (2) and (3),

$$
\begin{aligned}
e(X, Y) & \leqslant \frac{|X||Y| D}{n}+\lambda \sqrt{|X||Y|}=\frac{b t^{2} D}{n}+\lambda \sqrt{b} t=D t\left(\frac{b t}{n}+\frac{\lambda \sqrt{b}}{D}\right) \\
& \leqslant D t\left(b \varepsilon+\frac{\lambda \sqrt{b}}{D}\right)<D t(b \varepsilon+(1-b \varepsilon))=D t
\end{aligned}
$$

which is a clear contradiction.

Finally, suppose that $G$ fails to have property 2 from Definition 8 . Then there are sets $X, Y \subseteq V\left(G^{\prime}\right)$ with sizes $\varepsilon n$ and $\alpha n$ respectively such that $e(X, Y)=0$. But, by (2) and $(3)$,

$$
e(X, Y) \geqslant \frac{|X||Y| D}{n}-\lambda \sqrt{|X||Y|}=\alpha \varepsilon D n-\sqrt{\alpha \varepsilon} \lambda n>0 .
$$

Again, this is a contradiction.

\section{Proofs of Theorems 4, 5, 6 and 7}

Proof of Theorem 4. Let $\varepsilon_{0}:=\frac{\varepsilon}{4 d+2}$. By Theorem 15, (substituting with $\alpha:=\varepsilon / 2, b:=$ $d+1, \rho=\varepsilon_{0}$ and $\left.\varepsilon:=\varepsilon_{0}\right), G(n, c / n)$ almost surely contains a subgraph $G^{\prime}$ of order at least $\left(1-\varepsilon_{0}\right) n$, which is an $\left(\varepsilon_{0}, d+1, \varepsilon / 2\right)$-expander. By Corollary $9, G^{\prime}$ contains every tree with maximum degree $d$ and order

$$
\left(1-2 d \varepsilon_{0}-\varepsilon / 2\right)\left|V\left(G^{\prime}\right)\right| \geqslant\left(1-(4 d+1) \varepsilon_{0}\right) \cdot\left(1-\varepsilon_{0}\right) n \geqslant(1-\varepsilon) n .
$$

Proof of Theorem 6. Let $\varepsilon_{0}:=\frac{\lambda}{2 d}$. By Theorem 15, (substituting with $\alpha:=\varepsilon / 2, \rho:=$ $\min \left\{\varepsilon_{0}, \varepsilon / 2\right\}, \varepsilon:=\varepsilon_{0}$, and $\left.b:=d+1\right), G(n, c / n)$ almost surely contains a subgraph $G^{\prime}$ of order at least $(1-\rho) n$, which is an $\left(\varepsilon_{0}, d+1, \alpha\right)$-expander. By Corollary $10, G^{\prime}$ contains every tree $T$ with maximum degree $d$ and order

$$
(1-\varepsilon / 2)\left|V\left(G^{\prime}\right)\right| \geqslant(1-\varepsilon / 2) \cdot(1-\varepsilon / 2) n \geqslant(1-\varepsilon) n,
$$

provided that $T$ has at least $\lambda n$ leaves. 
Proof of Theorem 5. Let $\varepsilon_{0}:=\frac{\varepsilon}{4 d}$. By Theorem 16, (substituting with $\alpha:=\varepsilon / 2, b:=d+1$ and $\left.\varepsilon:=\varepsilon_{0}\right)$, every $(n, D, \lambda)$-graph $G$ is an $\left(\varepsilon_{0}, d+1, \varepsilon / 2\right)$-expander. By Corollary $9, G$ contains every tree with maximum degree $d$ and order $\left(1-2 d \varepsilon_{0}-\varepsilon / 2\right) n=(1-\varepsilon) n$.

Proof of Theorem 7 . Let $\varepsilon_{0}:=\frac{\lambda}{2 d}$. By Theorem 16, (substituting with $\alpha:=\varepsilon, b:=d+1$ and $\left.\varepsilon:=\varepsilon_{0}\right)$, every $(n, D, \lambda)$-graph $G$ is an $\left(\varepsilon_{0}, d+1, \varepsilon\right)$-expander. By Corollary $10, G$ contains every tree $T$ with maximum degree $d$ and order $(1-\varepsilon) n$, provided that $T$ has at least $\lambda n$ leaves.

Acknowledgements: Part of this project was done when József Balogh and Wojciech Samotij were supported by the Visiting Scholar Program of the Department of Mathematics at WKU. We would like to thank the anonymous referee for their valuable suggestions and comments.

\section{References}

[1] N. Alon, M. Krivelevich, and B. Sudakov, Embedding nearly-spanning bounded degree trees, Combinatorica 27 (2007), 629-644.

[2] N. Alon and J. Spencer, The probabilistic method. Third edition, John Wiley \& Sons, Inc., 2008.

[3] J. Balogh, B. Csaba, and W. Samotij, Local resilience of almost spanning trees in random graphs, submitted.

[4] J. Friedman and N. Pippenger, Expanding graphs contain all small trees, Combinatorica 7 (1987), 71-76.

[5] P. Haxell, Tree embeddings, Journal of Graph Theory 36 (2001), 121-130.

[6] M. Pei, List colouring hypergraphs and extremal results for acyclic graphs, Ph.D. thesis, University of Waterloo, 2008.

[7] L. Pósa, Hamiltonian circuits in random graphs, Discrete Mathematics 14 (1976), 359-364.

[8] B. Sudakov and V. Vu, Local resilience of graphs, Random Structures and Algorithms 33 (2008), 409-433. 